Instituto Internacional de Investigación y Desarrollo Tecnológico Educativo INDTEC, C.A.

DOI: https://doi.org/10.29394/scientific.issn.2542-2987.2017.0.0.4.61-81

OAI-PMH: http://www.indteca.com/ojs/index.php/Revista Scientific/oai

\title{
Fortalecimiento de la Historia Regional desde el Francés como Lengua Extranjera
}

Autoras: Carol del Carmen Terán González Universidad Nacional Experimental "Rafael María Baralt", UNERMB carolterang@gmail.com Trujillo, Venezuela Deysi Josefina Montilla Álvarez Universidad Nacional Experimental "Rafael María Baralt", UNERMB deysima 45@hotmail.com Trujillo, Venezuela

Roexana Andreina Castillo Vázquez Universidad de Los Andes Núcleo Universitario Rafael Rangel, NURR roexanacastillo90@gmail.com Trujillo, Venezuela

\section{Resumen}

El presente estudio tuvo como objetivo general analizar la historia regional desde el francés como lengua extranjera. La misma se llevó a cabo bajo la metodología de investigación descriptiva con la modalidad proyectiva desde un diseño de campo; para la realización de esta investigación se trabajó con la totalidad de los docentes de francés del nivel de educación Media General del municipio Valera, estado Trujillo los cuales conformaron una muestra de 16 docentes; su finalidad fue la de conocer cuáles eran los contenidos y estrategias que aplicaba el docente en el aula en el área de lengua extranjera, específicamente en francés, desde el conocimiento sobre sus orígenes e historia regional. Se pudo conocer que se puede establecer una articulación entre el área del francés con el área de historia desde la historiografía local.

Palabras clave: historia local; francés como lengua extranjera; enseñanza aprendizaje. 


\section{Strengthening of the Regional History from the French Idiom as a Foreign Language}

\section{Abstract}

The present research had as main proposal to analyze the regional history since the French idiom as a foreign language. This one, was worked under the methodology of descriptive research by the modality projective from a field design; for the making of this research the authors worked with all French idiom teachers of the ordinary middle school level in the municipality of Valera, Trujillo state; its goal was to let know which the contents that the teacher applied in the foreign language classroom were, and, specifically in French idiom, from the awareness about their origins and regional history. The investigators were able to know that an articulation between French area and history area could be given from the local historiography.

Keywords: regional history; french as a foreign language; teaching learning. 


\section{A manera de introducción}

El francés es un idioma que abre nuevos horizontes; por tal motivo, se ha visto la necesidad de fortalecer el proceso de formación de esta lengua extranjera desde la identidad propia del educando, diseñando e implementando distintas estrategias de enseñanza aprendizaje a través de un medio que ha sido parte de la globalización, el cual se ha convertido en muestra indispensable para la sociedad, es decir, los medios digitalizados, los cuales se han venido incorporando en el ámbito, no solo de educación, sino en general, tomando en cuenta que la gestión de conocimiento y las nuevas tecnologías han influido en todos los espacios del saber humano; por tal razón, el Sistema Educativo Bolivariano en su Currículo acopla como uno de sus ejes integradores Tecnología de la Información y Comunicación.

Es importante señalar que, una de las áreas que se puede encontrar en dicho currículo es el de Lenguaje, Comunicación y Cultura, el cual señala las lenguas extranjeras como parte del proceso pedagógico en uno de sus componentes y donde se da paso a la enseñanza aprendizaje de diversos temas para el desarrollo de las estructuras allí señaladas, pero que, en este proyecto se estudia el realce de la historia local como uno de esos temas, para así, integrar la asignatura de francés junto con el aprendizaje de la historia a partir de las nuevas tecnologías.

En la actualidad, el francés constituye un idioma de labor y una lengua oficial en la ONU, la Unión Europea, la UNESCO, la OTAN. Es la lengua extranjera que junto al inglés, representa el principal idioma hablado en los cinco continentes. Además, es el más aprendido después del inglés y la novena lengua más dialogada del mundo. Partiendo de ello, es importante señalar todos los vínculos que unen al ser humano con la sociedad, el lazo de la lengua es uno de los más fuertes y consistentes, ya que es a través de él que se funda el sentimiento de pertenencia a una comunidad. En el mundo actual, se favorecen cambios sociales, políticos y culturales, de allí la 
importancia que tiene dar a conocer lo local en distintos idiomas. Así lo plantea Cansigno, (2006:24)

La lengua francesa es la red precursora de una comunidad de 63 miembros asociados y observadores de la Organización Nacional de la Francofonía (OIF), y 28 países la tienen como lengua oficial. Asimismo, la lengua francesa es una de las dos lenguas oficiales del Comité Internacional Olímpico y la lengua oficial de trabajo de la mayoría de las organizaciones internacionales, la ONU y la Unión Europea (Cansigno, 2006: 24).

Por su parte, es oportuno señalar la historia local o regional en esta investigación, ya que la misma es aquella base cuyo legado hace que se le encuentre sentido a la cultura, a las cosas que se tienen, a lo que rodea al ser humano en el ambiente; es ese hecho que incide en el crecimiento integral como persona lleno de preguntas y dispuesto a crear memoria en los humanos; es el rescate de valores, de pertenencia e identidad cultural, se trata de darle importancia a esas casas antiguas, monumentos históricos, personajes solemnes de nuestra región, destacar la motivación que tienen todos los habitantes en hacer notar, valer y sentir la cultura de su región o localidad en pro de la defensa de los entes que hacen reflexionar.

Por la razón expuesta, vincular una lengua extranjera con la historia local resulta importante, más aún si se quiere visionar y resaltar los elementos culturales, históricos, así como también fortalecer la memoria colectiva de un estado o país a nivel internacional. No obstante, en Venezuela dicha vinculación es una mirada poco estudiada; además, es de hacer notar que el Sistema Educativo Venezolano ha venido incorporando en el currículo elementos sobre la historia local, el lenguaje y la interculturalidad en la educación convencional; empero, estos aspectos siguen mirándose de forma fragmentada y aislada. 
Partiendo de estos supuestos, es importante contextualizar que la historia local sigue siendo poco asumida en las instituciones educativas, no con esta acotación se pretende decir que no se enseña la historia, sino más bien, se enseña una historia fragmentada; así también, es primordial acotar que dentro de los nuevos tiempos educativos en Venezuela se viene dando una integración de áreas de conocimiento.

Por tal razón, resulta interesante que los docentes vinculen el área del lengua, cultura e idioma con la de ciencias sociales, tal como se pretende analizar en el presente trabajo, donde se busca integrar la asignatura de francés con elementos que involucran la historia de la localidad; así lo plantea el Diseño Curricular del sistema Educativo Bolivariano (2007: 64) citado por León (2009: 406) “...que deben transversalizar de manera armónica y holística, las áreas de aprendizaje y expresarse en los elementos que estructuran y condicionan el proceso de aprender en la escuela".

El conocer la historia de la localidad, del lugar donde se nace, donde nacieron valientes defensores de la patria y se efectuaron hechos históricos hace sentir aún más orgullosos de la tierra en que se nació y cultiva ese sentido de pertenencia que deberían tener todas aquellas personas ocupantes de su región. Es a través de la historia que, los pueblos seguirán existiendo a lo largo de la vida y deben ser los profesores, después de familiares, quienes deben transmitir y motivar a los aprendices de cada día a obtener conocimiento sobre la historia local.

En este sentido, para la integración de áreas desde la historia local a partir de la lengua del francés, es indispensable el uso de herramientas tecnológicas que alcancen las exigencias actuales de un mundo globalizado. El avance tecnológico brinda una facilidad de acceso a la información, puesto que muchas personas cuentan, no solo con equipos de alta tecnología, sino también con proyectos computarizados que acercan al investigador a 
información que se halla fuera de su contexto geográfico, como es el caso del proyecto o programa Canaima.

Es por esta razón que, se estudia las tecnologías de la información y la comunicación como una herramienta que sea de gran influencia, no solo para los estudiantes, sino para todas aquellas personas que quieran conocer un poco más de su cultura. Específicamente, un instrumento que permita divulgar la importancia del estado Trujillo en la historia venezolana, dicha región posee en su entorno una memoria histórica de gran importancia para el país.

En función de lo antes señalado, se origina la necesidad de efectuar la siguiente interrogante ¿Cómo incide el uso de una lengua extranjera en el fortalecimiento de la historia local del estado Trujillo?

\section{Propósito General}

Analizar la historia regional desde el francés como lengua extranjera en el municipio Valera, estado Trujillo.

\section{Recorrido teórico}

En este proceso de investigación, este apartado presentará las teorías que sustentarán el estudio, así como sus aportes para el desarrollo de la misma. Así también, es importante resaltar el enfoque de los temas que son parte de la estructura del contenido de este estudio, mediante el cual se podrá fundamentar y dar solidez al marco de referencia de la investigación.

\subsection{Aprendizaje Significativo}

Teóricos como Ausubel (1983: 136), enfatizan en que el aprendizaje implica una "reestructuración activa de las percepciones, ideas, contenidos y esquemas que el aprendiz posee en su estructura cognitiva"; asimismo, este concibe al estudiante como un "procesador activo de la información" y sostiene 
que el aprendizaje es sistemático y organizado, pues es un fenómeno complejo que no se reduce a simples asociaciones memorísticas.

David Ausubel propuso el término «Aprendizaje significativo» para designar el proceso a través del cual la información nueva se relaciona con un aspecto relevante de la estructura del conocimiento del individuo. A la estructura de conocimiento previo que recibe los nuevos conocimientos "concepto integrador». El aprendizaje significativo se produce por medio de un proceso llamado Asimilación. En este proceso, tanto la estructura que recibe el nuevo conocimiento, como este nuevo conocimiento en sí, resultan alterados, dando origen a una nueva estructura de conocimiento. (Osorio, 2007: 53).

La teoría propuesta por Ausubel ha tomado gran valor para la educación venezolana, siendo considerada como sustento teórico dentro del Currículo Bolivariano Nacional, por considerarla como una propuesta que junto con otras teorías (Psicogenética y socio cultural), contribuirían a la formación integral de estudiantes, esta teoría se caracteriza por dar valor y atender las diferencias individuales del estudiante, atendiendo a sus experiencias y haciéndolo participante activo del proceso educativo, por considerarlo en plena capacidad de construir su propio conocimiento.

Los materiales educativos computarizados responden a estas demandas de los estudiantes, ya que, pueden trabajar a su propio ritmo, y la variedad de elementos atiende a las diferentes formas de aprender, los que son más visuales, auditivos y hasta los kinestésicos, aunque de manera virtual. Atendiendo a los principios propuestos por esta teoría y la relación que establece entre aprendizaje y desarrollo, se pueden caracterizar sus fortalezas de la siguiente manera:

- El aprendizaje es personal, ya que depende de los recursos cognitivos del alumno, de sus experiencias, necesidades y expectativas. 
- La incorporación de nueva información a la estructura cognitiva del estudiante modifica la existente y lleva a la construcción de una nueva, a la reconstrucción de esquemas de conocimiento, es decir, se establece una relación entre los nuevos conocimientos y los ya adquiridos, lo que garantiza una retención más duradera de la información.

\subsection{Enfoque Socio-Cultural: Lev S. Vygotsky}

La teoría propuesta por Vygotsky también sustenta el Currículo Bolivariano Nacional, atendiendo a la importancia que esta asigna a las relaciones e intercambios sociales, donde el estudiante va más allá de la simple adaptación pasiva, este busca la evolución.

Una de las estrategias bandera de la educación venezolana es precisamente el trabajo cooperativo y colaborativo entre los estudiantes; se habla del convivir como elemento importante en la formación del Ser, colocando al docente como facilitador del proceso y al estudiante como participante en la construcción de su aprendizaje; concibiendo a la escuela como centro del quehacer comunitario y como espacio de comunicación (entre otros), sumando relevancia al contexto y a las relaciones de intercambio.

A partir de la tesis presentada por Vygotsky, Rodríguez (2004: 47), plantea: La formulación de la ley es clara con respecto a que el desarrollo cognoscitivo, emerge de la interacción social y de la mediación semiótica que caracteriza la vida en la sociedad. En el curso de las interacciones con las otras personas, el individuo entra en contacto y aprende a utilizar las herramientas (instrumentos y artefactos) y los sistemas simbólicos de su cultura (entre los que se encuentra el lenguaje) el aprendizaje estimula y activa una variedad de procesos mentales que afloran en el marco de la interacción con otras personas... 
Atendiendo a los aportes de la teoría de Lev Vygotsky a la educación y la relación que establece entre aprendizaje y la interacción con otros, se puede caracterizar:

- La Interacción y cooperación social es importante en la construcción de los aprendizajes y en desarrollo del estudiante.

- El hombre busca su evolución y bienestar, por lo que actúa sobre la realidad para adaptarse, para transformarla y transformarse a sí mismo.

- Da especial Importancia al contexto, ya que a partir del contacto con este se potencia su desarrollo y aprendizaje.

- Plantea la formación social de la mente, el convivir y el hacer, determinan el conocer.

- El estudiante puede lograr más con la ayuda de otros (nivel de desarrollo potencial) que por sí solo (nivel de desarrollo real).

- El estudiante construye a partir de su realidad; el entorno del estudiante influye en su personalidad y devenir, más aún, lo define.

Dada la influencia del entorno en la formación del valor referido a la identidad regional y nacional, adquiere importante anclaje la propuesta de Lev Vygotsky.

\subsection{Enseñanza de una lengua extranjera: mirado desde el Aprendizaje Integrado de Contenido y Lengua Extranjera}

Los estudios sobre el aprendizaje de una lengua extranjera han demostrado que, para lograr un buen nivel de competencia en ella, es necesario tener mucho contacto con el idioma de un modo natural. Los estudiantes necesitan poseer una senda espontánea a la comunicación dialogada, predilectamente dentro de un tejido interactivo en el que puedan obtener información sobre la estructura y el funcionamiento de la lengua extranjera. En tales circunstancias, los educandos pueden comprobar las 
suposiciones que se están constituyendo sobre el idioma que están aprendiendo. El aprendizaje de una lengua extranjera es un proceso largo y natural. El estudiante tiene que pasar ineludiblemente por los períodos del conocimiento "imperfecto" antes de dominar los diferentes aspectos de la lengua extranjera.

Por tal razón, el estudio a presentar se orienta hacia la enseñanza de un idioma extranjero de forma incidental, ya que el aprendizaje no se produce exclusivamente cuando se aplica consciente e intencionadamente a aprenderlo; sino que se produce cuando la atención de quien aprende está centrada en algo diferente y no en la lengua, como es el caso cuando el camino didáctico pretende enseñar contenido curricular a través de una lengua extranjera, aspecto que está marcado en el enfoque dentro de la investigación que se desarrolla, de allí que el enfoque utilizado sea Aprendizaje Integrado de Contenido y Lengua Extranjera (AICLE). Así lo plantea Marsh (2002: 18):

El Aprendizaje Integrado de Contenido y Lengua (AICLE) es un término genérico y se refiere a cualquier situación educativa en la que una lengua adicional $-\mathrm{y}$, consecuentemente no la más frecuentemente utilizada en el contexto- se usa para la enseñanza de asignaturas diferentes a la lengua (Marsh, 2002: 18).

Teniendo en cuenta lo antes expuesto, la idea preliminar, que exhortaba en que se trataba de un enfoque "de doble foco", para dar a entender el equilibrio existente entre contenido y lengua extranjera, en cuanto a relevancia que asume cada componente, más recientemente, y después de un tiempo de "rodaje" en las aulas, los expertos parecen haber reconsiderado los componentes clave del AICLE y reparado en el papel capital que representa en este enfoque las habilidades para aprendizaje (técnicas de estudio, destrezas de aprendizaje, estrategias de aprendizaje...) (learning skills), y ya se habla de un enfoque con tres enfoques o pilares -el contenido, la lengua 
extranjera y las habilidades para el aprendizaje-(Mehisto, Marsh y Frigols, 2008: 69), lo que da el régimen de la jerarquía que se le concede a estas últimas confrontadas en cuanto al estatus, al contenido y a la lengua extranjera.

\subsection{El francés como lengua extranjera}

En este apartado se hará un recorrido por el francés como lengua extranjera desde el año 1988, posteriormente a esa fecha, en todo caso, es difícil encontrarse explícitamente con esa expresión en los trabajos de investigación relativos al francés para fines específicos. En la actualidad, para este tipo de enseñanza se ha optado mayoritariamente por la denominación français sur objectif spécifique (francés para fines específicos). Es una fórmula poco original, puesto que, es una traducción literal de la expresión inglesa english for specific purposes.

Caracterizar esta metodología es una tarea ardua, en la medida en que se trata de una metodología abierta y sincrética, sobre todo a partir de la segunda mitad de la presente década. Sin embargo, de lo que no cabe duda es de que esta última etapa se caracteriza fundamentalmente por un conocimiento lingüístico de los discursos especializados (Jacobi, 1987) mucho más exhaustivo y por unas aplicaciones pedagógicas más certeras, ambos aspectos están, sin embargo, en constante evolución.

Estos son los rasgos que identifican a esta última etapa metodológica: primero, no se trata, como ya hemos adelantado, de una metodología cerrada sino abierta y enormemente ecléctica, si se utiliza el acertado concepto de Puren (1994: 7-17). Junto a eso, las múltiples especialidades, la complejidad del discurso en cuestión, los distintos perfiles de alumnos, los diversos niveles de motivación, el lugar que ocupa la asignatura en los planes de estudio en el caso de tratarse de enseñanzas regladas, entre otros, aconsejan liberalizar y flexibilizar en la medida de lo posible los cánones metodológicos; la figura del 
enseñante cobra fuerza puesto que, de este depende la confección casi a medida de los programas de enseñanza, la puesta en marcha de estos, así como el control de los resultados de los mismos.

Contrariamente a los comienzos en los que se desdibujaba la figura del profesor, centralizándolo casi todo en torno al estudiante, esta metodología se sustenta fundamentalmente en la labor particular de aquél; la tendencia a interpretar las enseñanzas especializadas y las generales como opuestas se desvanece. $\mathrm{Ni}$ las primeras deben circunscribirse al conocimiento de unas puntuales habilidades lingüísticas haciendo caso omiso de las particularidades extralingüísticas (Byram, 1992: 42; Zarate, 2003: 7) ni las otras han de asociarse a unos fines formativos sin especificar desligados de objetivos de comunicación concretos (Eurin y Henao, 1992: 70).

Todos los componentes del aparato pedagógico -la parcial identificación de las necesidades y los objetivos, los contenidos, el material de trabajo, las estrategias y técnicas de aprendizaje, la progresión, la evaluación, entre otros han de ajustarse al máximo a unos futuros e hipotéticos objetivos de comunicación oral y escrita, siendo el objetivo principal de estas enseñanzas la adquisición y uso de habilidades lingüístico-comunicativas, escritas u orales; es pertinente privilegiar métodos de trabajo y contenidos de base en consonancia con la dimensión discursiva de la lengua.

Esto implica considerar parámetros tan diversos de naturaleza lingüística y extralingüística, como la adquisición de los enunciados más corrientes de la lengua general, los conceptos y las construcciones discursivas propias del campo especializado o el conocimiento de las situaciones de comunicación profesional en las que presumiblemente el usuario tendrá que poner en práctica sus conocimientos de lengua extranjera. 


\subsection{La historia regional}

Para la historia venezolana, la historia regional representa un desafío; reto que abre puertas al estudio que se está generando en este boceto de trabajo, por lo cual definir la historia local es de relevancia, ya que esta concepción conforma el corpus teórico de la investigación; de allí que, para el mismo se tome la definición planteada por Medina (2005: 78):

La Historia regional es aquella que nos remite al pasado de las localidades, sean parroquias, barrios o urbanizaciones, ofreciéndonos un panorama de las motivaciones, individuales y colectivas, de un gran valor para el conocimiento, cultivo y uso de la población en general. Un rasgo característico de este tipo de historia es que se sirve de fuentes de tipo oral $y$, particularmente, provenientes de las comunidades, lo cual le confiere un contenido afectivo de gran valor por ser, precisamente, la experiencia subjetiva de sus habitantes. (Medina, 2005: 78).

La historia local fundamenta su definición en el descenso del nivel de reflexión de lo macro a lo micro, de lo global a lo local, en problemáticas y retos sociales que se asientan hacia el hombre y la mujer de carne y hueso, hacia los seres humanos y sus planes, hacia la gente de casa y sus maneras de pensar y de vivir. Es una historia que se sitúa al particular de las fronteras del espacio recurrible de las personas (barrios, veredas, corregimientos y municipios pequeños) y que converge con la memoria colectiva de la comunidad y de los sujetos sencillos y comunes que la componen: una historia que se sitúa en el universo inferior y microscópico de la sociedad, vivido por habitantes reales.

Así también es preciso señalar que, la pequeña historia es parte fundamental de la identidad del ser humano, por tanto, debe ser parte del sujeto de aprendizaje; todo estudiante de educación básica, media y 
universitaria debe tener una vinculación con la historia local, ya que ella forma parte de su identidad. Medina (2005: 51), plantea acerca del tema lo siguiente:

La importancia de la Historia Local reside en el hecho de ser fuente de "diagnóstico" de sucesos muy especiales para las comunidades que se traducen en un cúmulo de conocimiento afectivo capaz de fortalecer la identidad de la comunidad, la integración de sus miembros y las acciones tendientes al desarrollo de su entorno inmediato (Medina, 2005: 51).

Tomando en cuenta el enunciado anterior, la historia local forma parte del individuo desde su nacimiento, la misma es un legado que debe darse a conocer de generación en generación; esta fomenta y refuerza la identidad de los pueblos y de las naciones, por ello, su privilegio en el proceso de formación dentro del sistema educativo venezolana.

Por tal motivo, la historia local hoy está planteada como un instrumento pedagógico útil en ese encuentro que se plantea con la indagación histórica y social, que debe ser pensado desde la educación básica y media, como una oportunidad de formación y de acercamiento hacia un pensamiento más crítico, un conocimiento más real e interactivo dentro de las instituciones educativas y la sociedad en general.

\section{Recorrido Metodológico}

En la realización de esta investigación se estableció la estrategia metodológica descriptiva. En consecuencia, el marco metodológico de la presente investigación alude al momento técnico presente en todo proceso de investigación; donde es necesario situar al detalle, el conjunto de métodos, técnicas e instrumentos que se emplearán en el proceso de recolección de los datos requeridos en la investigación, con la finalidad de cumplir con el objetivo general de la investigación. 


\subsection{Tipo de investigación.}

De acuerdo al problema planteado y en función de sus objetivos, se incorporó el tipo de investigación denominada descriptiva, de la cual se entiende por Sabino (2000: 60) como "La descripción de las características fundamentales del conjunto homogéneo del fenómeno utilizando criterios sistemáticos que permitan poner de manifiesto su estructura o comportamiento". Así entonces, en esta investigación los autores describieron características o propiedades del grupo estudiado. Respecto al nivel del estudio, el autor citado indica que esto se refiere al grado de profundidad con que se aborda un objeto o fenómeno. Indicando si se trata de una investigación exploratoria, descriptiva o explicativa. El presente trabajo es descriptivo por cuanto caracterizó a grupo con el fin de establecer su estructura o comportamiento, en este caso los docentes de la institución estudiada.

\subsection{Diseño de la investigación.}

Una vez definido el tipo de estudio, el investigador debe concebir la manera práctica y concreta de responder a las preguntas de investigación. Esto implica seleccionar o desarrollar un diseño de investigación y aplicarlo al contexto particular de su estudio. En tal sentido, se hace necesario señalar que el diseño se refiere al plan o estrategia concebida para responder a las preguntas d investigación. Por tanto, indica al investigador lo que debe hacer para alcanzar sus objetivos de estudio, contestar la interrogante que se ha planteado. Se basa en el apoyo que orienta la investigación sobre el sustento minucioso de un diseño documental y de campo, el diseño de la investigación es la estrategia general que adoptan los investigadores para dar respuesta a la problemática planteada. 


\section{Resultados de la investigación}

Para la obtención de datos que pudieran dar respuesta a los objetivos planteados en esta investigación, se aplicaron encuestas estructuradas a los 16 docentes quienes fueron objeto de estudio. Dicha encuesta contó con 16 ítems o preguntas relacionados a la enseñanza en el área de francés y el área de historia en la búsqueda de fortalecer la identidad regional o local desde una lengua extranjera.

Los resultados permitieron observar que los docentes de francés del municipio Valera, estado Trujillo no incluyen en sus clases contenidos que accedan a estudiar la historia e identidad local desde la lengua extranjera. Esta información acotada por los encuestados deja ver la falta de iniciativa en los docentes de incluir las áreas de conocimiento en el aula de clase tal como lo pide el currículo bolivariano en sentido de brindar una mejor calidad educativa a los estudiantes venezolanos en pro de una formación integral.

\section{A manera de Conclusión}

Durante la investigación, se pudo determinar que a los educandos se les ha abarrotado de datos, fechas y héroes militares; sin embargo, se ha obviado a algunos hombres del pueblo y personajes civiles propios de la historia local y regional; en este orden de ideas, es importante señalar que no se hace énfasis en la historia de la región, la que pertenece a las localidades, en la cual los trujillanos han crecido, y a la que todos deberían tener más acceso, ya que, es a partir de lo pequeño que se da paso a la gran historia de un país o nación.

En este apartado, se aprecia que la historia local da paso a la construcción sociohistórica de la nación; en ella se ve construida la identidad, los valores, costumbres, tradiciones y hechos que conforman arquitectónicamente un país; es ella la que muestra la gran historia detrás del otro, ese pasado que formó el camino al presente y que se guarda en la 
memoria colectiva del individuo. Tomando en cuenta estos aspectos, surge la idea de proyectar la historia local, no solo en las localidades, comunidades y regiones venezolanas, sino arriesgarse en mostrar la "historia pequeña", esa historia propia de comunidades y localidades en el idioma español, aventurarse a presentarla mediante una lengua extranjera, es decir, el francés como herramienta de enseñanza de la historia regional.

Puesto que, uno de los elementos que ha permitido la difusión y reconocimiento de hechos y personajes históricos ha sido la expresión oral; la oralidad es uno de los mecanismos de construcción y transmisión de costumbres y tradiciones; legado promovido con la participación de los ancianos, las ancianas, los sabios y las sabias de la comunidad por ser ellos quienes, a través de sus vivencias, rescatan el valor histórico de su localidad, a diferencia del docente quien centra su enseñanza únicamente en el estudio mecánico del francés, sin tener en cuenta que los estudiantes viven en un espacio específico y que estos adquieren un aprendizaje significativo a partir de temas relacionados con su propia identidad.

\section{Referencias}

Ausubel, D. (1983). Psicología educativa. Segunda Edición. México: Editorial Trillas.

Byram, M. (1992). Culture et éducation en langue étrangère. Didier, París. Cansigno, Y. (2006). El francés, lengua de comunicación internacional. Reencuentro, núm. 47, diciembre, pp. 23-28.

Eurin, S. y Henao, M. (1992). Prácticas de francés científico. Hachette/Aupelf.

Jacobi, D. (1987). Textes et images de la vulgarisation scientifique. Berne, Eds. Peter Lang. 
León, A. (2009). Estructura y base conceptual del diseño curricular del sistema Educativo Bolivariano. Revista EDUCERE, Año 13. № 45, ISSN: $1316-4910$.

Marsh, D. (2002). Bilingual Education \& content and Language Integrated Learning. International Association for Cross-Cultural Communication, Language Teaching in the Member States of the European Union (Lingua) University of Sobornne, París.

Medina, A. (2005). Manual de historia local. Biblioteca Nacional.

Mehisto, P., Frigols, M. y Marsh, D. (2008). Uncovering, CLIL. Oxford: Macmillan.

Osorio, N. (2007). La teoría crítica de la sociedad de la escuela de Frankfurt: algunos presupuestos teórico-críticos. Revista Educación y Desarrollo Social, pp. 104-119.

Puren, Ch. (1994). La didactique des langues étrangères à la croisée des méthodes. Essai sur l'éclectisme. Paris: Didier-Crédif.

Rodríguez, P. (2004). La teoría del aprendizaje significativo. [Artículo en línea]. Recuperado de: http://cmc.ihmc.us/papers/cmc2004-290.pdf

Sabino, C. (2000). El Proceso de la Investigación. Editorial Panapo. Venezuela.

Zarate, G. (2003). Las competencias interculturales: del modelo teórico al diseño curricular. Universidad Antonio de Nebrija, Madrid. 


\section{Carol del Carmen Terán González}

e-mail: carolterang@gmail.com

Nacida en la ciudad de Valera Edo Trujillo, Venezuela.

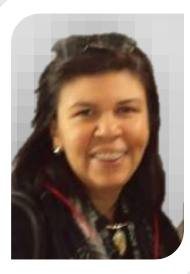
Cursó estudios de Doctorado en Educación en la Universidad Rafael María Baralt, posee Maestría en Docencia para la Educación Superior en la misma universidad, Maestría en Literatura Latinoamericana en la Universidad de los Andes y Licenciatura en Educación Mención Castellano y Literatura ULA-NURR investigadora del Centro de investigaciones literarias y lingüísticas Mario Briceño Iragorry. (CILL) ULANURR, Jefe de la unidad de investigación y proyecto de la Casa de Historia Trujillo, articulista e investigadora con 14 años de servicio en el área de educación y literatura. Ponente en eventos nacionales e internacionales. Docente invitada en pregrado y Postgrado, ULA, UNERMB, UNESR. Coordinadora de la Línea de investigación Memoria, Educación y Discursos emergentes (UNERMB), miembro de la Línea de investigaciones Ciudadanía, hermenéutica y proyectos sociales. (UNERS). Ha realizado artículos en distintas revistas arbitradas del país. 


\section{Deysi Josefina Montilla Álvarez}

e-mail: deysima 45@hotmail.com

Nacida en Valera, estado Trujillo. Licenciada en

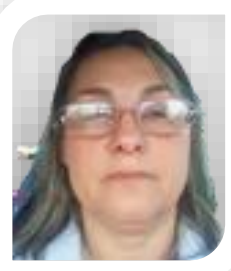

Educación. Tiene maestría en Administración de la

Educación Básica, UNERMB. Ha trabajado como docente por más de quince años en el Ministerio del Poder Popular para la Educación. Doctorante de la Universidad Nacional Experimental "Rafael María 
Roexana Andreina Castillo Vázquez

e-mail: roexanacastillo90@gmail.com

Nacida en Valera, estado Trujillo, Venezuela.

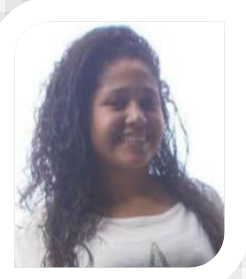

Estudiante de educación mención Lenguas Extranjeras del Núcleo Universitario "Rafael Rangel" ULA. Fue preparadora en el área de francés por dos (2) años. Ha participado en numerosos eventos de investigación en distintas áreas como ponente, comité organizador y asistente. Adscrita al Centro de Investigaciones Lingüísticas y Literarias "Mario Briceño Iragorry" ULA-NURR. Miembro de la línea de investigación Memoria, Educación y Discursos emergentes (UNERMB). Miembro del Taller "AbyaYala" NURR.

El contenido de este manuscrito se difunde bajo una Licencia de Creative Commons ReconocimientoNoComercial-Compartirlgual 4.0 Internacional 